\title{
ПРОБЛЕМЫ ПОСТРОЕНИЯ ДОКСАСТИЧЕСКИХ МОДЕЛЕЙ
}

\section{Н.П. Козаченко}

Одной из важных методологических процедур, осуществляемых в процессе познания, является построение модели исследуемой области. Как в процессе рассмотрения уже готовой модели, так и собственно в самом ходе ее построения, исследуемые объекты представляют для изучения все новые нюансы и аспекты, требующие более глубокого анализа и уточнения. Модель в данном случае представляет собой некий эксперимент, манипулирующий воображаемым объектом. Идеализации замещают реальный объект в целях его изучения и помогают увидеть сущность предмета исследования посредством наблюдения и изучения его существенных связей и отношений. Исследование реального процесса зачастую настолько затруднено, что упрощенное идеализирование кажется необходимым и закономерным.

Процесс изменения знаний содержит в своей основе сложнейший механизм, который связывает воедино различные сферы деятельности человека. K тому же он столь быстротечен и изменчив, что ухватить реальный момент единственного изменения практически невозможно. Модель изменения знаний - это структура, воспроизводящая действительный процесс в упрощенной схематизированной и наглядной форме. Попытка исследовать именно логическую составляющую динамики изменения знаний и приводит нас к возможности построения доксастических моделей.

Данная работа представляет собой продолжение нашей предыдущей статьи [2]. Мы рассмотрим некоторые наиболее специфические аспекты, которые должны учитываться при построении адекватной доксастической модели. Прежде всего, это возможность принимать во

Актуальні проблеми духовності

(Відп. ред.: Я.В. Шрамко)

Кривий Ріг (2007), 245-258 
внимание дополнительные познавательные ограничения, которые чаще всего нехарактерны для естественного процесса изменения знаний, но необходимы для построения управляемой модели.

\section{1 Какие познавательные ограничения мы учитыва- ем при построении моделей?}

Предыдущие проблемы моделирования систем изменения знаний и убеждений касались основных вопросов построения моделей и ограничений, коими мы должны оперировать ради достижения максимальной приближенности к реальным процессам. Еще один вопрос, касаемо ограничений можно сформулировать и таким образом, - какие именно ограничения, кроме конечности или исчислимости множества убеждений мы можем реализовать при построении модели. И дело не только в том, каким образом мы должны представить эти ограничения, а в том, собственно о каких ограничениях идет речь. В данном контексте рассмотрения речь идет об отличиях модели знаний, которыми оперирует машина и которыми оперирует человек. Ведь практически не существует ситуации, в которой процесс познания осуществляется в рамках той или иной модели, представленной сейчас в эпистемологии. Да, эти модели представляют собой идеализации и значительно упрощены, но не приводит ли такого рода упрощение к искажениям в представлении самого процесса. Мы ограничиваем познавательные способности субъекта, оперирующего знаниями, налагая условие конечности множества его убеждений. Это кажется интуитивно понятным, потому что странно было бы предположить возможность человека «ворочать» бесконечным набором убеждений. Но действительно конечен ли этот набор? Мы говорим о парадоксе «всезнания» как о следствии логической замкнутости человеческого набора убеждений. Но на самом деле мы скорее искусственно создаем это ограничение, для того чтобы облегчить жизнь машине, которая могла бы смоделировать процесс изменения человеческого знания. Но если по совету Винера попробовать оставить «человеку - человеческое, вычислительной машине - машинное» $[1$, с. 22]. И отвлёкшись от модели, от любого механистического представления системы убеждений, мы можем заметить, что человек, даже не осознавая бесконечность своих убеждений, прекрасно нею пользуется, при необходимости осуществляя нужные выводы и дополнения. По словам Хайдеггера, человек должен «в уже домысленном высматривать непомысленное, которое все еще скрыто 
внутри уже помысленного» [3], а сколько этого недомысленного знания содержится в том количестве информации, которое хранится в наших убеждениях. Кажется, существенным здесь есть различие между оперированием и осознанием. И хотя Герденфорс говорит о принципиальном отказе от всяческого сравнения с носителем знания, мы не можем упустить тот факт, что субъект, оперирующий знаниями играет немаловажную роль в отражении динамики их изменения. В данном случае речь идет именно о человеке как носителе знаний, потому что осознание характерно как раз для него. Конечно, попытка моделировать процесс осознания уведет исследователей в решение вопросов, связанных не только с эпистемологической проблематикой. Поэтому очень приемлемым есть подход, в котором используется разделение убеждений на основное множество убеждений и базу убеждений, которая по необходимости и пополняется недостающими, неосознаваемыми ранее высказываниями. K базе не применимы ни требование последовательности, ни требование замкнутости относительно логического следования. Основные познавательные операции производятся на каркасе множества убеждений и потом влекут за собой изменения в базе. Именно к основному множеству убеждений мы имеем право выдвигать требования рациональности познающего субъекта. Но возникает еще один вопрос, каким образом мы выделяем это основное множество, на котором и производим операции. Для его ограничения необходимо задействовать время. Таким образом, мы приходим к понятию эпистемического состояния субъекта, как к тому основному множеству убеждений, которые в данный момент он принимает, а с точки зрения логического подхода это не что иное как множество высказываний, которые субъект считает истинными.

\section{2 Как связаны между собой исправление (восста- новление) и операторы изменения и взаимовыра- зимы ли они?}

В большинстве моделей принимаются к рассмотрению только внешние влияния, которые и приводят к изменению системы убеждений. При этом, модели, ассимилирующие вход не рассматривают изменения, происходящие внутри системы убеждений. Они показывают эффекты влияния внешних причин на системы, которые изменяются только в ответ на такие внешние влияния (входы), а иначе устойчивы. Это делает их более-менее подходящими для представления важных 
аспектов изменения состояний человеческого разума и состояний убеждений. По крайней мере, для большинства целей, это разумная идеализация. Такой подход допускает игнорирование изменений в убеждениях человека, которые не имеют никакой прямой внешней причины, чтобы лучше сосредотачиваться на механизмах изменения, вызванного извне. Но, сколь мы уже затронули вопрос о наличии базы убеждений, которая проецируется в состояние убеждений, то стоит рассмотреть и возможный результат такой проекции. Исходя из того, что сама база может содержать скрытое противоречие, можно предположить, что состояние убеждений также может оказаться противоречивым. Таким образом, не имея внешнего входа, мы получаем необходимость консолидации уже имеющихся убеждений. Такая ситуация не редкость и требует способа преодоления противоречия. Кроме того, схожая ситуация может возникнуть и в результате выполнения некоторой операции, обусловленной внешним входом и тогда возникает необходимость повторения (итерации) познавательных действий для разрешения возникшей проблемы. Возможны два варианта элиминации противоречия.

(1) Изменение. Первый предполагает действия, аналогичные которым осуществляет модель, получившая внешний вход. Таким образом, изменение системы убеждений будет происходить по ранее установленному образцу, рассматривая противоречие как внешне поступившую информацию.

(2) Восстановление (исправление). Второй подход предполагает возможность внутреннего решения проблемы. Система предпринимает действия, которые оставляют конфликт нерешённым, но вместо этого делает необходимый выбор для восстановления непротиворечивой информации системы.

В модели AGM, действие исправления по возможности упрощено это просто оператор идентичности. Фактически система возвращается к предыдущему непротиворечивому состоянию. Действия изменения в AGM более сложны. В других моделях, с более сложным действием исправления, могут быть достаточно просто реализованы действия изменения. Фактически между обоими этими действиями существует заметная связь. Они оба направлены на устранение противоречия, оба предполагают возможность выбора. Отношения между исправлением и изменением должны исследоваться с формальной и философской точки зрения. Мы не знаем, действительно ли эти подходы существенно различны или один из них может быть сведён к другому. 


\section{3 Возможна ли «чистота» познавательных опера- ций}

Вопрос определения познавательных действий разработан довольно тщательно. На данный момент мы имеем несколько основных операций, которые с небольшими вариациями используются в моделях изменения знаний. В модели AGM есть три типа изменения убеждений. Основная идея происходит от более ранней работы Айзека Леви [11] и [12]. Это сокращение, расширение и ревизия (пересмотр). В сокращении указанное предложение (представление убеждений) должно быть удалено из набора убеждений, без добавления чего-либо к нему. Это зафиксировано постулатом включения $(\mathbf{K} \div a) \subseteq \mathbf{K}$ и говорит о том, что результат сокращения всегда должен составлять подмножество исходной системы убеждений. Другими словами, после выполнения операции сокращения путем удаления какого-то высказывания, мы не должны обнаружить в полученном множестве убеждений новое высказывание, которого до удаления там не было. Следовательно, сокращение множества убеждений посредством нетавтологии $a$ в результате даст новый набор убеждений, который не содержит $a$, а так же не содержит никаких добавленных высказываний. Фактически, таким определением мы «очищаем» операцию сокращения от других познавательных операций. В этом смысле мы постулируем «чистое» сокращение.

Расширением предполагается, что указанное предложение добавляется теоретико-множественным способом также без удаления чего-либо. Расширенное множество замкнуто относительно логического следствия. Пересмотром предполагается, что указанное предложение добавлено к набору убеждений, при условии, что полученный набор убеждений непротиворечив и замкнут относительно логического следования [4]. То есть операция добавления также постулируется в чистом виде.

Обе эти операции в их чистом виде можно назвать первичными. Так как ревизия определима в терминах сокращения и расширения с помощью равенства Леви или Харпера. Эта операция представляет собой последовательное применение сокращения и расширения. Вначале следует произвести поиск высказываний, противоречащих добавляемому и удалить их, а затем добавить новое высказывание. Если мы добавим высказывание $a$ в систему, которая содержит $\sim a$, полученное множество убеждений окажется противоречивым и выполнение операции замыкания превратит его в бесконечное множество всех 
формул. Таким образом, операцию ревизии можно определить следующей формулой $\mathbf{K} * a=(\mathbf{K} \div \sim a)+a$, которая и получила название равенства Леви. Для наборов убеждений, которым появление противоречий не страшны, например для баз убеждений, для ревизии можно использовать равенство Харпера, которое предполагает другой порядок выполнения действий сокращения и расширения. Но по сути все изменения убеждений в модели AGM строятся с помощью двух первичных операторов. Здесь также возникает вопрос-существуют ли указанные операции в чистом виде. И если относительно расширения и ревизии этот вопрос не слишком актуален, то применимо к сокращению он требует определенного решения. Как уже было сказано, согласно традиционному определению, результатом сокращения есть подмножество первоначального множества убеждений, которое не содержит исходное предложение. Следовательно, это - действие, в котором старые убеждения удалены, но никакие новые убеждения не добавлены. Очень трудно найти пример «чистого» сокращения, в котором не добавлено никакое новое убеждение. Скорее всего, когда мы удаляем убеждение, мы делаем это потому, что мы получили какое-то новое убеждение, которое исключает старое.

Для конкретности приведём пример, указанный Ханссоном, предположим, что ранее я верил в то, что динозавры исчезли в результате внезапного климатического изменения $(a)$. И только намного позже один геолог сказал мне, что это - только одна из нескольких конкурируюших гипотез. Это заставляет меня отказаться от моего убеждения $(a)$ без того, чтобы верить в его отрицание. Строго говоря, это не пример «чистого» сокращения, так как новое убеждение было принято к сведению как одно из несколько конкурирующих научных гипотез относительно исчезновения динозавров. В литературе по динамике убеждений, примеры вроде этого часто интерпретируются как случаи «чистого» сокращения. Новым убеждением, коим собственно и вызвано сокращение, пренебрегают, и не включают в новое множество убеждений. Это - неточное, но удобное соглашение, позволяет более легко находить примеры сокращения. Мы иногда гипотетически отказываемся от некоторого убеждения, чтобы принять противоречащее убеждение. Такие гипотетические сокращения, или сокращения для пользы аргумента, иногда принимают за «чистые» сокращения. Однако, эта интерпретация сомнительна, поскольку в действительности эти действия не предприняты субъектом. Вообще чистое сокращение может быть задумано как операция, которая имеет место на определенной части набора убеждений, например на той, которая не содержит 
никаких убеждений, относящихся к другим убеждениям или их подтверждающих.

Трудность «чистого» сокращения не должна повлечь поверхностное рассмотрение этой операции. Более того, сокращение - существенный элемент рационального изменения убеждений. Обычно оно задействовано как часть более сложных изменений, которые влекут и потерю, и приобретение информации. Для формального анализа, полезно рассмотреть модели «чистого» сокращения. Однако, это все еще оставляет открытым вопрос, о том как иметь дело с действиями, которые интуитивно приемлемы как сокращения, хотя они не есть «чистыми» сокращениями, так как сопряжены с добавлением новых убеждений.

\section{4 Синтаксическая независимость моделей. Реали- зация нелингвистической информации}

Кроме уточнения познавательных операторов и их идеализаций, интересные замечания рождаются при рассмотрении области их применения, а так же возможности их применения и полученных результатов. В этом ключе следует отметить, что в AGM все познавательные действия производятся над высказываниями. Но некоторые исследователи подвергают сомнению единственность этого подхода. Макеш Далал [5] предложил принцип синтаксической независимости системы. Фактически это означает отказ от приоритета высказываний при изменении множества убеждений, потому что результат изменения не зависит в данном представлении от синтаксической формы принятой либо имеющейся информации. В фактических системах изменения убеждений, отдельное предложение может и не быть носителем информации, дополняющей его логическое содержание. В AGM и схожих системах такое невозможно, так как они рассматривают только изменения с помощью высказываний. Синтаксически разные наборы убеждений обычно рассматриваются как информационно отличные друг от друга, но они могут нести одну и ту же информацию. Следовательно, статически эквивалентные базы убеждений могут иметь различные динамические свойства при изменении [8]. Некоторые авторы довольно неохотно учитывают больше чем одну базу убеждений с тем же самым логическим замыканием. Однако, только учитывая такую возможность, можно использовать выразительную силу баз убеждений, большую чем множеств убеждений. В модели AGM это учитывается при сокраще- 
нии наборами предложений, так называемое «множественное сокращение». Сокращение различными наборами с тем же самым логическим замыканием может давать различные результаты [7].

В программировании воздерживаются от использования предложений или наборов предложений, дабы не представить больше, чем подразумевало бы их соответствующее логическое содержание, что в потенциале возможно в любой модели на основе языка. Независимость синтаксиса поэтому должна рассматриваться не как критерий адекватности для моделей на основе языка обработки информации, а скорее как свойство, которое появляется в некоторых, но не во всех процессах идеализации, использованных для построения моделей.

Но если мы примем что входы для сокращения, расширения и пересмотра должны быть предложениями в формальном языке, как и представленные убеждения, то увидим, что это не создает никаких проблем. Обычно эпистемический субъект чаще всего стремится изменить свои убеждения с помощью нелингвистических входов, например сенсорных впечатлений. Модели изменения убеждений, которые работают с высказываниями, предполагают, что все входы, воздействующие на состояния убеждений, могут быть соответственно представлены предложениями. Когда я слышу шум дождя на улице (сенсорный вход), я регулирую мое состояние убеждений, то есть изменяю его таким образом, чтобы включить высказывание «На улице идет дождь» (лингвистический вход). Следовательно, в AGM и сродных системах, каждое действие изменения связано с предложением одним из трёх способов: сокращение, расширение или пересмотр посредством опять же предложений. Кроме того, уникальность AGM подхода состоит в том, что для каждого типа действия существует точно одно действие для каждого предложения (в действительности для каждого класса логически эквивалентных предложений), а это значит, что есть точно одно сокращение для каждого предложения и т. д.

\section{5 Проблема разложения}

В предыдущих разделах мы рассмотрели проблему единственности представления убеждений и возможность работы с познавательными действиями в чистом виде. Но относительно познавательных операций возникает еще один вопрос, - могут ли указанные традиционные действия охватить весь спектр изменений, которые претерпевает множество убеждений. Существует специфическая сфера исследований, 
основанная на предположении возможности представления других типов изменения, кроме представленных в AGM. Фундаментальное предположение о динамике изменения убеждений, представленное Айзеком Леви [11] состоит в том, что сложные изменения могут быть проанализированы как последовательности простых изменений. Исходя из вышесказанного, опишем принцип разложения, сформулированный Фурманом.

Каждое правильное изменение убеждений может быть представлено как последовательность сокращений и расширений [6].

Принцип разложения не должен восприниматься как действительное требование изменять убеждения пошаговым способом - одно расширение или сокращение единовременно, но оговаривается, что результаты комплексного изменения должны быть такими же, как и при пошаговом применении заявленных операций.

Исследования в этой сфере дали возможность предложить несколько дополнительных категорий действий. В многократном сокращении и многократном пересмотре, исходное множество состоит скорее из наборов предложений, чем из отдельных предложений. Действия нераспределённой по приоритетам ревизии отличаются от обычной ревизии тем, что исходное высказывание не всегда принимается. Действия по консолидации увеличивают степень целостность множества убеждений, делая их последовательными или согласованными. Только что было упомянуто «смешанное» сокращение, также возможны и другие смешанные действия. Ввиду этого разнообразия, проблема разложения множественного действия на основные или атомарные типы действия стоит отдельного исследования. Исходя из такой универсальной постановки проблемы, можно сформулировать следующий вопрос, существуют ли атомарные познавательные действия, в терминах который можно представить все возможные изменения множества убеждений.

\section{6 Разложение классической операции ревизии}

Более подробно остановимся на разложении такого комплексного действия как ревизия. В отличии от предложенных в предыдущем параграфе, способ ревизии множества убеждений достаточно хорошо описан в литературе и фактически представляет собой общепринятый подход. Две главных задачи оператора ревизии $*$ состоят в том, чтобы добавить новое убеждение к множеству убеждений $K$, и гарантиро- 
вать непротиворечивость конечного множества убеждений $\mathbf{K} * a$ (при условии непротиворечивости $a$ ). Первая задача может быть выполнена путём расширения $\mathbf{K}$ за счёт $a$. Вторая задача может быть выполнена предварительным сокращением на его отрицание $\sim a$. Если набор убеждения не влечёт $\sim a$, тогда $a$ может быть добавлено без потери непротиворечивости. Поэтому оператор ревизии может быть определён через два первичных действия таким образом:

(1) Сокращение посредством $\sim a$.

(2) Расширение посредством $a$.

Или в более кратком изложении: $\mathbf{K} * a=(\mathbf{K} \div \sim a)+a$ (равенство Леви).

Выполнение этих действий в обратном порядке невозможно если $\mathbf{K} \cup\{a\}$ противоречиво, тогда $K+a$ всегда будет одним и тем же (a именно, идентичным всему языку), таким образом будут потеряны все начальные значения.

Точно так же как соответствующие операторы для множеств убеждений, оператор ревизии для баз убеждений может быть построен с помощью применения двух первичных действий. Чтобы ревизировать множество убеждений посредством $a$, мы производим расширение с помощью $a$ и сокращаем на $\sim a$. Именно в этом случае можно выполнять эти два действия в любом порядке - согласно равенству Леви или в обратном порядке:

(1) Расширение посредством $a$;

(2) Сокращение на $\sim a$.

Более сжато, это выражено обратным равенством Леви или, как его еще называют, равенством Харпера, $-\mathbf{B} * a=(\mathbf{B}+a) \div \sim a$.

Двум операторам ревизии, которые могут быть разложены с помощью равенства Леви (для состояния убеждений) или равенства Харпера (для баз убеждений) могут соответствовать различные формальные свойства [10]. Они соответствуют различным интуитивным представлениям о том, как преобразующая убеждения информация должна быть представлена в результате рационального познания субъекта.

При рассмотрении вариантов разложения ревизии интересно что последовательность множества убеждений сохраняется на каждом шаге сокращение + расширение, но существует промежуточное нефиксированное состояние, когда субъект не убеждён ни в самом высказывании $a$, ни в его отрицании $\sim a$. А в самом осуществлении комбинации 
действий расширение + сокращение, существует противоречивое промежуточное состояние множества, в котором присутствуют оба убеждения $a$ и $\sim a$, какой выбор должна осуществить модель? Которое из двух действий более вероятно? Наши интуитивные представления об этом, скорее всего будут различны в разных случаях. Рассмотрим примеры, приводимые Ханссоном.

(1) Энтони и Беатрис-супруги. Я всегда думал, что они оба принадлежат к Римской Католической церкви. Но однажды я услышал, как Беатрис сказала: «То, что мы принадлежим к различным вероисповеданиям никогда не создавало нам проблем в семье». Когда я услышал это, я отказался от убеждения, что Беатрис католичка, но сохранил убеждение, что Энтони католик (так как я видел как он несколько раз посещал местную католическую церковь).

(2) Когда Джозеф Блак узнал результатов нового эксперимента Лавуазье, он отказался от своего убеждения о флогистонной теории горения, и принял теорию кислорода Лавуазье.

(3) Я полагал, что Джон умер. Но затем я встретил его на улице [9].

В первом случае расширение + сокращение кажется наиболее приемлемым. В более общем виде: если очевидно, что новая информация должна быть принята, но не так очевидно, какое предыдущее убеждение она должна вытолкнуть, тогда комбинация расширение + сокращение кажется наиболее близкой к фактическому психологическому процессу.

Во втором случае не было стадии колебания, которая предполагала было бы принятие самого убеждения и его отрицания, сокращение + расширение ближе чем расширение + сокращение к этому виду преобразования убеждений.

В третьем случае трудно определить наиболее адекватную модель. Интуитивно, эти два действия кажутся одновременными-особенность, которую нелегко охватить в логическом представлении.

Таким образом, нас интересует вопрос, какова роль промежуточного состояния множества, которое предполагает его несогласованность и какие действия может повлечь такое состояние. 


\section{7 Разложение нераспределенной по приоритетам ревизии}

Отдельно рассмотрим вопросы, касающиеся разложения для нераспределённой по приоритетам ревизии убеждений. Под нераспределённостью по приоритетам, подразумевается неуверенность субъекта в предложенной для ревизии информации. Полученный внешний вход может быть ценным для субъекта и тогда он решит его принять, а может оказаться и ненужны, тогда он будет отвергнут. Обычная ревизия оперирует входящими высказываниями, которые имеют уже определенный приоритет и поэтому они принимаются без вынесения решения об их ценности. Кроме ценности получаемой извне информации учитывается и ее внутренняя непротиворечивость и согласованность с уже имеющимися убеждениями. Нераспределённая по приоритетам ревизия не гарантирует обязательного принятия во множество убеждений высказывания, которое ревизирует систему. Один из способов построения такого действия базируется на следующем двушаговом процессе решение + пересмотр (decision + revision $)$ [8]. Вначале мы решаем, принять или отклонить входящее высказывание. После этого, если входящее высказывание было принято, включаем в множество убеждений посредством (обычной) ревизии.

То есть мы имеем такой алгоритм:

(1) Решить, должен ли вход быть принят или отклонен.

(2) Если вход был принят, произвести ревизию посредством $a$.

Другой подход к нераспределённой по приоритетам ревизии убеждений состоит в том, чтобы временно принять новую информацию и, если это приводит к несогласованности, впоследствии восстановить её отвергая или входящее убеждение или некоторые из предыдущих убеждений, - расширение и консолидация (expansion + consolidation) [8]. Алгоритм действий такой:

(1) Расширение посредством $a$.

(2) Консолидация множества убеждений.

Здесь консолидация - это процедура, которая делает множество убеждений согласованным. Но этот подход был развит только для баз убеждения. Консолидация может быть определена как удаление 
противоречащих высказываний. Другой интересный путь развития заключается в использовании «ограниченного» оператора консолидации, который объединяет только подмножества баз убеждений. Вопреки полной консолидации, этот процесс не уничтожает всю несогласованность. Это довольно реалистичная особенность, так как в реальной жизни несогласованности часто допускаются и, будучи локальными, не размножаются вплоть до вырождения состояния убеждений.

\section{8 Итог: возможность построения более реалисти- чной модели изменения убеждений}

Исходя из предыдущей и настоящей статьи, можно выделить и систематизировать основные проблемы построения моделей изменения убеждений. Эти проблемы могут быть разделены на две основных категории. K первой категории можно отнести общие вопросы, возникающие при моделировании процесса изменения и непосредственно наиболее реалистичном и удобном представлении динамики изменения убеждений. Это такие вопросы как: выбор типа модели; выбор уровня идеализации; определение ограничений, налагаемых на модель; объектный язык в целом; форма представления убеждений, а также условных и модальных предложений; возможность реализации различных степеней убеждений; удобное представление структуры состояния убеждений и установление связей в нем, и некоторые другие.

Вторая категория вопросов предполагает тщательное рассмотрение операций, их реализацию и свойства внутри модели. К этой категории будут отнесены вопросы о возможности применений познавательных операций в чистом виде, повторяемости познавательных операций, определении атомарных операций и дальнейшем их использовании в разложении комплексных операций. Также следует обратить внимание на возможность существования других познавательньх операций, отличных от классических и на удачные способы их представления.

\section{9 Литература}

[1] Винер Н. Творец и робот. - М., 1966.

[2] Козаченко Н.П. Модели изменения убеждений: некоторые открытые проблемы // Актуальні проблеми духовності-3б. на- 
укових праць / Вип. 7.-Кривий Ріг: Видавничий дім, 2006. C. 218-230.

[3] Хайдеггер M. Что значит мыслить? // Хайдеггер М. Разговор на проселочной дороге.-М., 1991.

[4] Alchourron C., Gardenfors P., Makinson D. On the logic of theory change: partial meet contraction and revision functions // Journal of Symbolic Logic. - 1985. - Vol. 50. - P. 510-530.

[5] Dalal M. Investigations into a theory of knowledge base revision: preliminary report // Seventh National Conference on Artificial Intelligence (AAAI-88). - 1988. - P. 475-479.

[6] Fuhrmann A. Reflective modalities and theory change // Synthese. 1989. - Vol. 81. - P. 115-134.

[7] Fuhrmann A., Hansson S.O. A survey of multiple contraction // Journal of Logic, Language and Information. - 1994. - Vol. 3. - P. 3976 .

[8] Hansson S.O. Taking belief bases seriously // Prawitz D., and Westersthol D. (Eds.) Logic and Philosophy of Science in Uppsala. Uppsala: Kluwer Academic Publishers, 1994. - P. 13-28.

[9] Hansson S.O. Ten philosophical problems in belief revision // Journal of Logic and Computation. - 2003. - Vol. 13(1). - P. 37-49.

[10] Hansson S.O. Reversing the Levi identity // Journal of Philosophical Logic. - 1993. - Vol. 22. - P. 637-669.

[11] Levi I. Subjunctives, dispositions and chances // Synthese - 1976. Vol. 34. - P. 423-455.

[12] Levi I. The Enterprise of Knowledge.-Cambridge, Massachusetts: The MIT Press, 1980. 